\title{
Linked Data-based Slide Repository: The Episodic Slide Retrieval Using the Episodic Keyword Networks
}

\author{
Tomohiro Iwasa, Yudai Kato, Shun Shiramatsu, Tadachika Ozono and Toramatsu Shintani \\ Department of Computer Science and Engineering, Graduate School of Engineering, Nagoya Institute of Technology, Gokiso-cho, \\ Showa-ku, Nagoya, Aichi 466-8555, Japan
}

\begin{abstract}
This paper focuses on developing a system that allows presentation authors to effectively retrieve presentation slides for reuse from a large volume of existing presentation materials. We assume episodic memories of the authors can be used as contextual keywords in query expressions to efficiently dig out the expected slides for reuse rather than using only the part-of-slide-descriptions-based keyword queries. As a system, a new slide repository is proposed, composed of slide material collections, slide content data and pieces of information from authors' episodic memories related to each slide and presentation together with a slide retrieval application enabling authors to use the episodic memories as part of queries. The result of our experiment shows that the episodic memory-used queries can give more discoverability than the keyword-based queries. Additionally, an improvement model is discussed on the slide retrieval for further slide-finding efficiency by expanding the episodic memories model in the repository taking in the links with the author-and-slide-related data and events having been post on the private and social media sites.
\end{abstract}

Key words: Slide retrieval, linked data-based slide repository, episodic keyword networks, linked data, episodic memories, social media, life event.

\section{Introduction}

Our goal in this study is to create a new presentation slide management method and a system to support the presentation authors with easily finding out the reusable presentation slides that have been created before for new presentation opportunities. Reuse of the existing presentation slides is commonly accepted in authoring the new presentation and alleviates the time and effort rather than cases to create the presentation slides from zero. Actually, individuals, teams and groups such as laboratories, and enterprises frequently create new presentation slides based on the existing presentation slides. For example, some researchers reuse one's own existing presentation slides and others reuse some other's presentations. According to an online survey, 105 out of 108 survey participants (i.e. 97\%) are reported on the reusing presentation content [1]. However, finding the desired presentation slides from a large number of existing presentation materials stored in the file servers is often a hard work especially for authors in a group at a large organization that may maintains collections of hundreds of presentations [2].

To address the desirability in effectively finding the reusable presentation slides, we propose to make use of the episodic information as cues in the presentation slide search process because the episodic memories of the authors that have been associated with each slide would be easily recalled rather than the specific keywords being contained in the presentation slide materials. Concretely, we construct Episodic Keyword Networks in the form of an episode-based relational data model and slide contents, composed of slides, episodic information such as matters relevant to the subject of slides to be reminded, some personal life events or social events occurred when the slides were created, and their links.

We call such links as Linked Data. According to the the Linked Data community web site http://linkeddata.

Corresponding author: Tomohiro Iwasa, Ph.D. Candidate. 
org/, the definition of Linked Data is a data form using the Web to connect related data that was not previously linked, or using the Web to lower the barriers to linking data currently linked using other methods, and a term used to describe a recommended best practice for exposing, sharing, and connecting pieces of data, information, and knowledge on the Semantic Web using URIs and RDF.

The key of the above method is that we use the episodic memories as part of keyword queries to retrieve the desired slides, thus, we call this new search method using Episodic Keyword Networks as Episodic Slide Retrieval.

Episodic Slide Retrieval is the practice we propose for Episodic Keyword Networks, based on the context search over the episode information in addition to the slide contents, gives more discoverability we assume in the search capability than the keyword-based query for the slide content descriptions.

We also provided the Linked Data-based Slide Repository as an implementation model of Episodic Keyword Networks in a form of the document repository for Episodic Slide Retrieval.

For authors' operation, our proposing browser-based search application of Episodic Slide Retrieval on the top of the Linked Data-based Slide Repository of Episodic Keyword Networks supports the slide-page-level search in the query operation, as well as the entire presentation material-level finding search option. The slide-page-level search option is prepared for authors in case the searched presentation has many slides like 50 or 100 pages, without manual finding efforts authors can further find the desired slides from that presentation efficiently. As a use case model to design, develop and evaluate in this study activity, we assume that about ten members belonging to the group that is supposed to use the Linked Data-based Slide Repository system, having provided a web application for sharing the Microsoft PowerPoint-based presentation among the group.

Additionally, an improvement model is discussed on the slide retrieval for further slide-finding efficiency by expanding the episodic memories model in the repository taking in the links with the author-and-slide-related data and events having been post on the private and social media sites.

We give the answers to the following study questions in this paper. (1) Is Episodic Slide Retrieval based on Episodic Keyword Networks effective? (2) What type of the query method is appropriate for information retrieval using episodic information? (3) What is the way to collect the piece of episodic information? (4) What is the implementation method for the system that support the slide reuse?

This paper is organized as follows. We present the details of Episodic Keyword Networks for the presentation slide reuse in Section 2, Linked Data-based Slide Repository in Section 3, Implementing Linked Data-based Slide Repository in Section 4, Evaluation in Section 5, Discussion in Section 6 and Conclusion in Section 7.

\section{Episodic Keyword Networks for Slide Reuses}

In this section, we discuss difficulties with reuse of the existing presentation slides and effectiveness of using the episodic information to find out the desired slides. Then, we propose Episodic Keyword Networks of Structuring Episodic Information as Linked Data.

\subsection{Difficulties with Slide Reuse}

In document retrieval, a full-text search is often used as a traditional technique for searching documents. However, there are cases when authors cannot the retrieve desired materials with the full-text search for presentation reuse. For example, it happens when authors cannot remember the full-text, including whether the characters are upper-case or lower-case, that are contained in a slide or slides. In fact, Ravasio et al. [3] have reported that remembering the full-text contained in desired documents is difficult for authors. 
Also, limitation of the keyword-based search in the contents of presentation slides is often experienced by authors. For example, a too-short query string can make the return with too many search results for authors. On the other hand, a very specific but inappropriate query key possibly makes no search results. Especially for authors, it is not easy to recall the appropriate keywords for query.

Moreover, some slides do not contain much information in the form of text because we have to arrange presentation elements such as text, shapes, and images in a limited space of the slide. As the first place of the ideal presentation design, the length of text on the slide is made as much shorter as possible because authors should make text letters larger to be easily seen by presentation audiences. Also, if the text length on the slide is too long, it is hard for presentation audiences to read and understand the description on the slide within a limited time.

Additionally, while authors may remember visual elements (e.g. shapes, images, layout, etc.) other than text, it is difficult to retrieve slides including visual elements by using full-text or keyword-based queries especially for slides that have a small number of or non text on them.

Therefore, in this study, we focus on the episodic information as extended keyword queries to utilize so as to efficiently find out the desired slides from the existing presentation materials.

\subsection{Using Episodic Information for Episodic Slide Retrieval}

In episodic memories, there are various types of memories, such as spatiotemporal (e.g. date and place), perceptual (e.g. visual and sound), semantic (e.g. what an event is and why an event occurs) and emotional information (e.g. positive or negative), that can be cues for prompting retrieval of the desired slides. Temporal and spatial information and perceptual detail rather than feelings are better recalled as those are perceived events [4]. Life-log images promote more genuine, detailed recall, whereas locational information promotes inferencing [5].

A user study illustrated that time and location serve as valuable contents [6]. From the studies above, query keys that are easily recalled will have benefits for authors to be used to retrieve the desired slides efficiently.

Then, the question for us is what kind of query is suitable for this purpose? There has been a prior work on using activity history and creation/access times for supporting personal information search [7]. Authors have event memories such as activity history, creation time and study activity as episodic memories. For example, authors may associate a slide with a submitted paper or an event (e.g. a conference, seminar, etc.).

Additionally, authors also have memories of text and shapes that the authors wrote or drew as episodic memory about authoring presentation materials. The recall of visual elements (existence of graphics, tables, and colors within the document) is easily made for authors than the recall of keywords of meaningful words within the document [8].

Hence, visual elements are also to be used for slide retrieval in our study. Tanaka et al. [9] proposed a slide retrieval method that considers shapes and arrangements of shapes, and the area ratio of the shape in a slide, by calculating similarities between a query and each shape in presentation slides.

The above shape-based method is an effective search method for the slides that contain shapes, but it is time-consuming to make queries composed of shapes in advanced because of complexity of their expressions for authors.

We present the slide retrieval method based on Episodic Keyword Networks, that are Network composed of slides, episodic information and the links between them. The episodic information is information associated with authoring slides and a presentation events (e.g. when and where the presentation was given, who authored the slides, what the author was 
doing when authoring the presentation, etc.). For example, the slide retrieval method based on Episodic Keyword Networks has ability to retrieve the slides with queries of the titles of the songs that the author was listening to when authoring the slides.

Also, we propose the attributed information related to the direct information from authors' or others' related posts in the social networking that will be part of the episodic information model.

\subsection{Structuring Episodic Information as Linked Data}

We construct Episodic Keyword Networks as Linked Data because interoperability of Linked Data makes Episodic Keyword Networks sharable, extensible, and easily reusable, and it is possible to model information propagation in the Network for slide retrieval.

Fig. 1 shows the structure of Episodic Keyword Networks.

A prefix "ressup" is an abbreviation for RESearch SUpport Platform.

In the following, we omit "ressup". ":Activity" is a class that represents study activity, and has "dc:created" property that represents the creation date, "dc:creator" that represents a creator of activity and ":event" property that represents an event such as a conference and a seminar where the user gave a presentation. ":Publication" is a subclass of ":Activity" and represents a product such as a paper, a presentation material and a study note. ":Paper" and ":Presentation Doc" are subclasses of ":Publication". “:Paper" represents a paper and ":Presentation Doc" represents a presentation material.

Since a paper has a relationship of the reference with a material that a user presented, ":Presentation Doc" has a "dc:reference" property that represents reference. ":PresentationDoc" also has ":composedOf" properties which represent inclusion relationships between presentations and slides. "Slide" is a class that represents a slide and has ":composedOf" properties and "reuse" properties. The ":composedOf" properties represent inclusion relationships between slides and presentation elements.

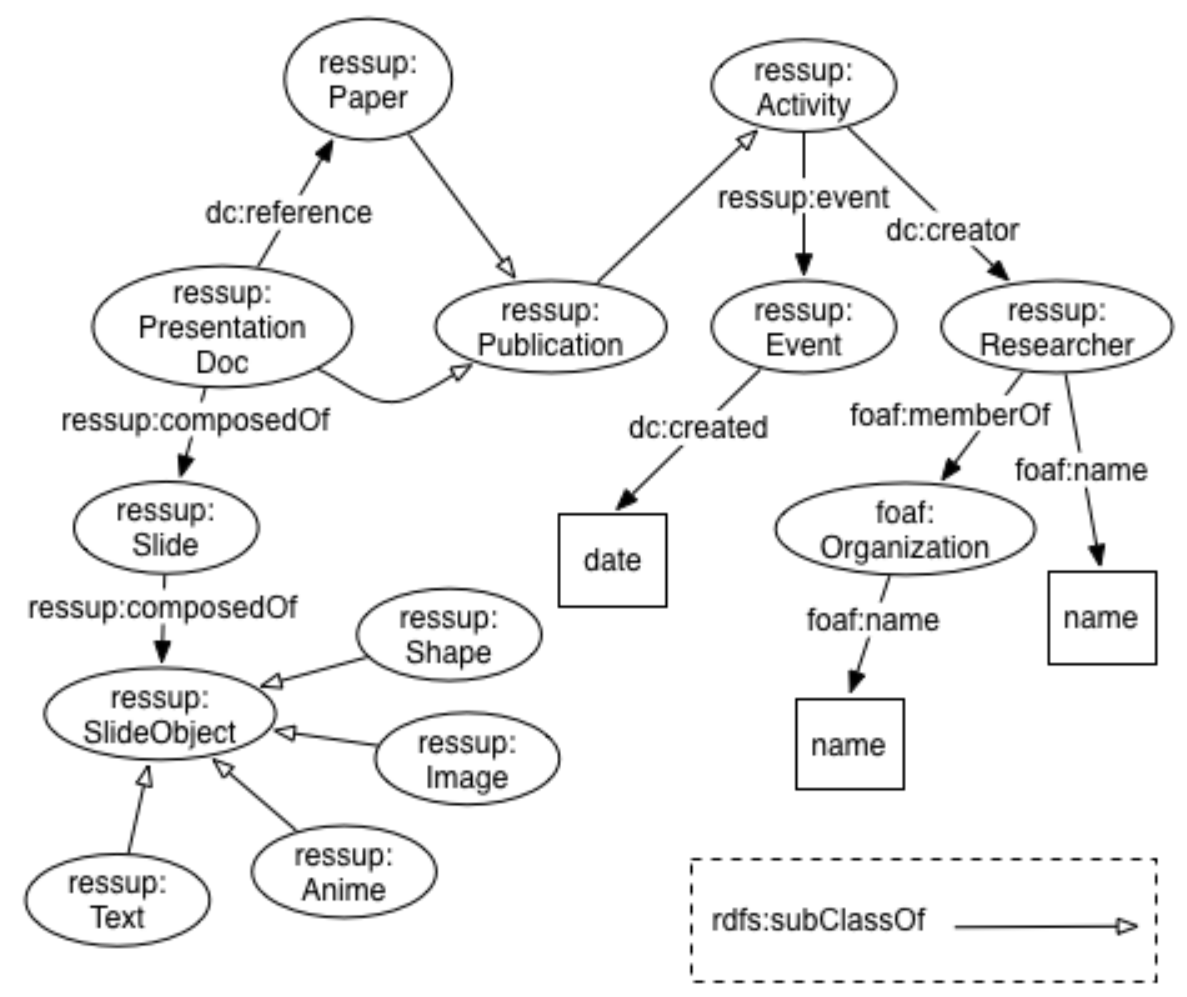

Fig. 1 Episodic Keyword Networks. 
The "reuse" property represents that a slide reuses another slide. A domain of ":composedOf" is the union of ":PresentationDoc" and ":Slide", and a range of ":composedOf" is the union of "Slide" and ":SlideObject". "SlideObject" is a class that represents a presentation element and has subclasses such as ":Text", ":Shape", ":Image", and ":Anime". ":Shape" corresponds to a shape such as a circle, a rectangle, a line and an arrow. ":Anime" represents an animation within the slides.

The "reuse" properties are useful for improving search precision. Reused slides may be reused again, that is, the reused slides are appropriate ones because they have been once selected for authors' needs. It is possible to change the ranking of search results by assigning higher weights to the reused slides. The ranking leads to improving the search precision.

\section{Slide Repository}

\subsection{Design Goals}

We identified the following design goals for a system to facilitate the slide retrieval functions for reuse of the presentation slides. (1) Supports the presentation slide reuse for authors in a group at a large organization that may maintains collections of hundreds of presentations. (2) Supports Episodic Slide Retrieval based on Episodic Keyword Networks. (3) Supports to find the desired slides put of a presentation material once that material has been identified.
In support of these goals, we have further identified the several key design features. (1) Provides a Web-based application that allows authors to share slides easily in the group the authors belong to. For a problem that the web browser do not display the slides in the PowerPoint format in a standard feature of the web browser itself, to address this issue, Linked Data-based Slide Repository has embedded a module to convert the Power Point files into the SVG format files. (2) Supports for constructing Linked Data for the desired slides from Linked Data proceedings in SPARQL.

Supports a user interface making authors easier to visually check and compare the retrieved slides.

\subsection{System Architecture}

Fig. 2 shows the architecture of Linked Data-based Slide Repository. The Linked Data-based Slide Repository has two main subsystems: at the front end containing the user interface of the Linked Data-based Slide Repository, and at the back end a transfer module, a search module, MySlideStack module and a presentation module. The user interface of the Linked Data-based Slide Repository supports all user interactions, receiving information that authors input to pass them to the back end modules as well as displaying the results from the Linked Data-based Slide Repository for authors. The convert module receives PowerPoint files uploaded by authors and information about author names and event names

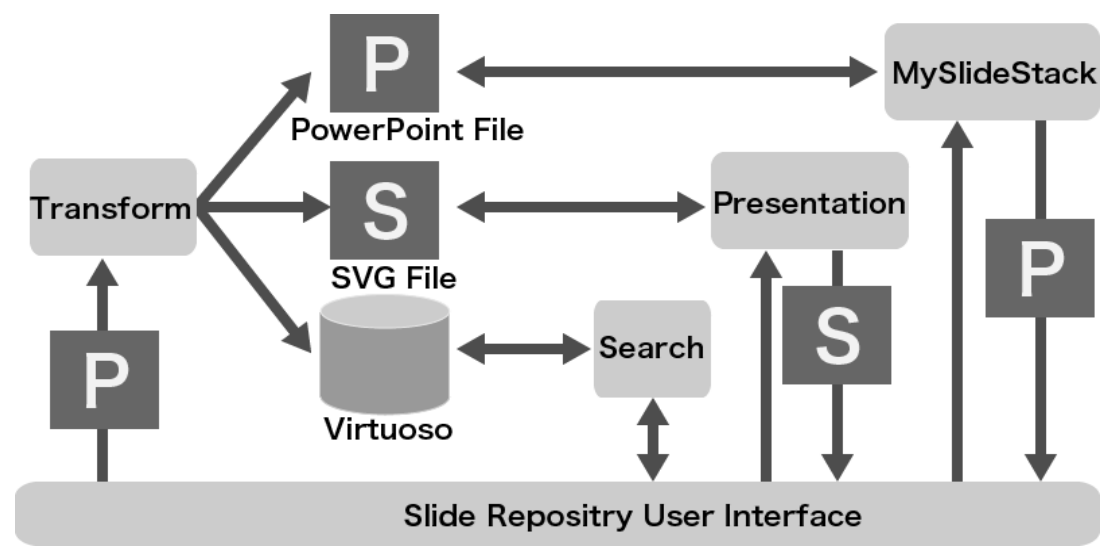

Fig. 2 System Architecture. 
inputted by the authors. The module, then, transforms the PowerPoint files into SVG files to construct Liked Data of the PowerPoint files and stores the PowerPoint files, the SVG files and Linked Data. The search module receives the text strings for the slide retrieval entered by authors from the user interface, then, transforms the text strings into queries in the SPARQL, that executes the queries over Virtuoso to receive the URIs of found slides from Virtuoso. Virtuoso is a multi-model server and utilized for multi-purposes (e.g. relational data management, RDF data management, Linked Data server, etc.). The version of the Virtuoso in Linked Data-based Slide Repository is 6.1. The MySlideStack module has two functions: a registration function and a download function for presentation slide materials. The registration function temporarily registers slides that have been retrieved from Linked Data-based Slide Repository in the search module as a result of authors' search operation. The download function downloads the PowerPoint files that have been registered in the registration function and authors searched for reuse. The presentation module allows authors to give a presentation using SVG files in Linked Data-based
Slide Repository on the web browser that authors will use at a presentation opportunity. In the process for reuse, authors register the desired slides in MySlideStack module, and combine the registered slides with the desired slides, and then, download the combined slides as one new PowerPoint file.

\subsection{Query Language}

$\mathrm{RDF}$ is a directed, labeled graph data format for representing information in the Web. SPARQL contains capabilities for querying required and optional graph patterns along with their conjunctions and disjunctions [10]. We use SPARQL to construct Episodic Keyword Networks and retrieve slides with it. SPARQL queries have capability to retrieve slides by using the episodic information.

\subsection{Examples of Episodic Keyword Networks}

Fig. 3 shows three examples of Episodic Keyword Networks. The round nodes represent URIs of the data and the rectangular nodes represent xsd:string. The light gray nodes are the desired slides and the dark gray nodes are cues for retrieval. We assume that a user searches slides containing some rectangles. The

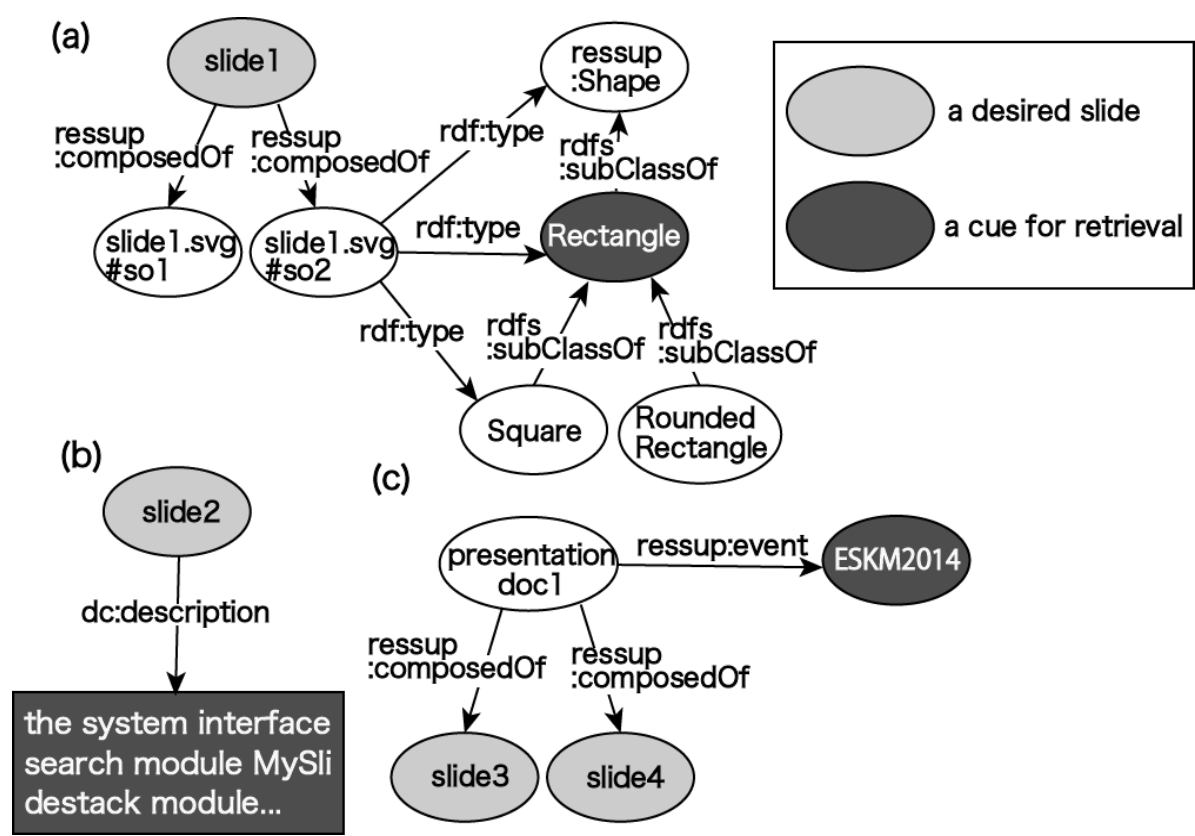

Fig. 3 Episodic Keyword Networks (a) search using a shape, (b) search using keywords, and (c) search using an event. 
right nodes ("ressup:Shape", "Rectangle", "Square" and "Rounded Rectangle") compose an ontology of shapes, and each node is owl:Class. A "Rectangle" class is a subclass of a "ressup:Shape" class that has shape classes (e.g. Circle, Line, Triangle, Arrow, etc.). "slide1.svg\#so1" and "slide1.svg\#so2" are presentation elements within "slide1". "slide1.svg\#so1" has "rdf:type" links to shape classes which represent that the presentation element is a square, a rectangle and a shape. In the process of search using a shape ("Rectangle"), the system search "slide1" by tracing all paths from "Rectangle" class to slides ("rdf:type" and "ressup:composedOf"). This example is an example of keyword-based search. We assume that a user searches slides containing a keyword ("interface"). "slide2" contains text that is "the system interface search module MySli..." and linked to the text with "dc:description". In the process of search using keywords, the system search "slide2" by using pattern is matching with a keyword ("interface"). We assume that a user searches slides within presentations given in ESKM2014. The "presentationdoc1" is composed of two slides and has a "ressup:event" link to "ESKM2014". In the process of search using an event "ESKM2014", the system searches "slide3" and "slide4" by tracing all paths from "ESKM2014" to slides ("ressup:event" and "ressup:composedOf”).

\section{Implementation}

In this section, we present a slide transformer, constructions of Episodic Keyword Networks, SPARQL queries and the user interface. Linked Data-based Slide Repository transforms the text strings receiving from authors into the SPARQL queries to be used for searching for slides in the SPARQL.

We present how to describe the SPARQL queries searching for slides in examples of Fig. 3. In the user interface, we show one example of the Linked Data-based Slide Repository user interface and present how to search for slides with queries such as keywords, events, authors and shapes.

\subsection{Slide Converter}

There are two problems with a web application supporting Episodic Keyword Networks and Linked Data-based Slide Repository. Firstly, Episodic Keyword Networks as Linked Data is composed of a set of links. The links are represented in the form of the subject-predicate-object expression. These expressions are known as triples in the RDF terminology. A subject must be always in the form of URI. Then, it is necessary to give URIs to presentations, slides, events, presentation elements, authors, and so forth. We can give a URI to each presentation material (a PowerPoint file) itself, but cannot give URIs to each slide pages of a PowerPoint file. Secondly, web browsers do not have a capability in a standard feature to display slides in the PowerPoint format.

Because of these reasons, we convert the PowerPoint files into the SVG format files. The number of the SVG files is equal to the number of pages of a presentation material. The created SVG slides are able to play animations (e.g. slide-in, bound, etc.). We can give a URI to a slide what is representing the entire slides separately as the SVG files. A presentation element is represented as a tag

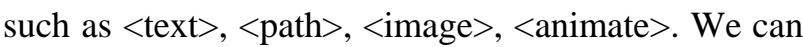
also give a URI to a presentation element by applying an id attribute to the tag in the xml structure. For example, if an id attribute of an element in a SVG file whose URI is "http://example.com/slide.svg" is "id1", a URI of the element is "http://example.com/slide. svg\#id1".

\subsection{Constructing Episodic Keyword Networks}

Linked Data-based Slide Repository updates Episodic Keyword Networks when authors upload the PowerPoint files from an upload form on the user interface of Linked Data-based Slide Repository. Then, the authors input author names and event names into 
the form to add more information on the PowerPoint file that has been uploaded. Linked Data-based Slide Repository analyzes the files and input data, and creates SPARQL queries based on the information by the result of analysis, and updates Episodic Keyword Networks by sending the queries to Virtuoso. We utilize the Apache POI API1 to analyze the files and get information about presentations, slides, presentation elements, and so forth. The Apache POI API provides pure Java libraries for reading and writing files in Microsoft Office formats, such as Word, PowerPoint and Excel. Table 1 shows the number of entities of Episodic Keyword Networks. There are 214 presentations and 3,102 slides (about 14 slides per a presentation material on average). Linked Data-based Slide Repository collects slides by uploading PowerPoint files from the upload form of Linked Data-based Slide Repository.

\subsection{SPARQL Queries}

Fig. 4 shows three queries for examples shown in Fig. 3. SPARQL queries allow the user to abbreviate URIs by declaring "prefix" abbreviations at line 1 of (1), (2) and (3). The "select" query form is used to get a list of URIs, that satisfy the pattern-matching requirements specified in the query. If a string is preceded by a question mark, it is treated as a variable that can represent a URI or a literal.

(1) The query gets URIs of slides that contain "Rectan-gle" as shapes. "?slide rdf:type ressup:Slide." represents that "?slide" is a slide at line 3. "?object0" represents a presen- tation element, which corresponds to "slide1.svg\#so1" and "slide1.svg\#so2" in Fig. 3. A "Rectangle" class is defined as a URI ("http://example.com/resources/Shape/Rectangle").

"ressup:numberOfShapes" represents the number of shapes in the slide at line 5 .

(2) The query gets URIs of slides that contain "interface" as text. The type of "?content" is xsd:string at line 6. "?content" corresponds to "the system interface search module..." in Fig. 3.
Table 1 The number of entities in slide stack.

\begin{tabular}{ll}
\hline & The number of Entities \\
\hline Presentations & 214 \\
Slides & 3,102 \\
Events & 49 \\
Authors & 16 \\
Shapes & 14,370 \\
Images & 2,585 \\
All & 166,153 \\
\hline
\end{tabular}

(3) The query gets URIs of slides within presentations given in "ESKM2014". Slide retrieval using events is approximately equivalent to slide retrieval using shapes. "ESKM2014" is defined as a URI ("http:// example.com/resources/Event/ESKM2014") like the shape of line 5.

\subsection{User Interface}

Fig. 5 shows a search interface for Linked Data-based Slide Repository.

Authors begin with accessing to the web page of Linked Data-based Slide Repository using the web browser. Authors search for slides by inputting keywords into a search form (Fig. 5(a)) and selecting elements from the drop-down lists (Fig. 5(b)) in the middle of the page. Fig. 5 shows the search results (Fig. 5 (c)) when a user searched slides with selecting both "Oval" and "Arrow" in the shape drop-down list. The user interface supports visual comparison of the slides that are displayed on the Web Application for authors as thumbnail images from the SVG slides. Authors can start to give a presentation with the SVG files by clicking the thumbnails.

Linked Data-based Slide Repository allows authors to search for slides with both full-text search and search inputting text strings of episodic information. The search form is for full-text search, and the drop-down lists are for search using episodic information. The drop-down lists have three types (a shape list, an event list and an author list).

The shape list contains names of shapes (e.g. "Square", "Oval”, "Column", "Right Arrow", "Down Arrow", etc.) within slides on Linked Data-based Slide Repository, 
(a)

1: prefix ressup: <http://example.com/ressup/ressuponto.owl\#>

select distinct ?slide where

?slide rdf:type ressup:Slide.

?slide ressup:composedof ?object 0 .

?slide ressup:numberofShapes ?num.

?object0 rdf:type <http://example.com/resources/ Shape/Rectangle>.

(b)

1: prefix ressup: <http://example.com/ressup/ressuponto.owl\#>

select distinct ?slide where

filter (regex (?content, 'interface','i'))

?slide rdf:type ressup:slide.

?slide ressup:numberOfShapes ?num.

?slide dc:description ?content.\}

(c)

: prefix ressup: <http://example.com/ressup/ressuponto.owl\#>

select distinct ?slide wheref

?slide rdf:type ressup:slide.

?slide ressup: numberofshapes ?num.

?pd ressup:composedof ?slide;

ressup:event <http://example.com/resources/Event/ ESKM2014>.

Fig. 4 Queries for the examples in Fig. 3.

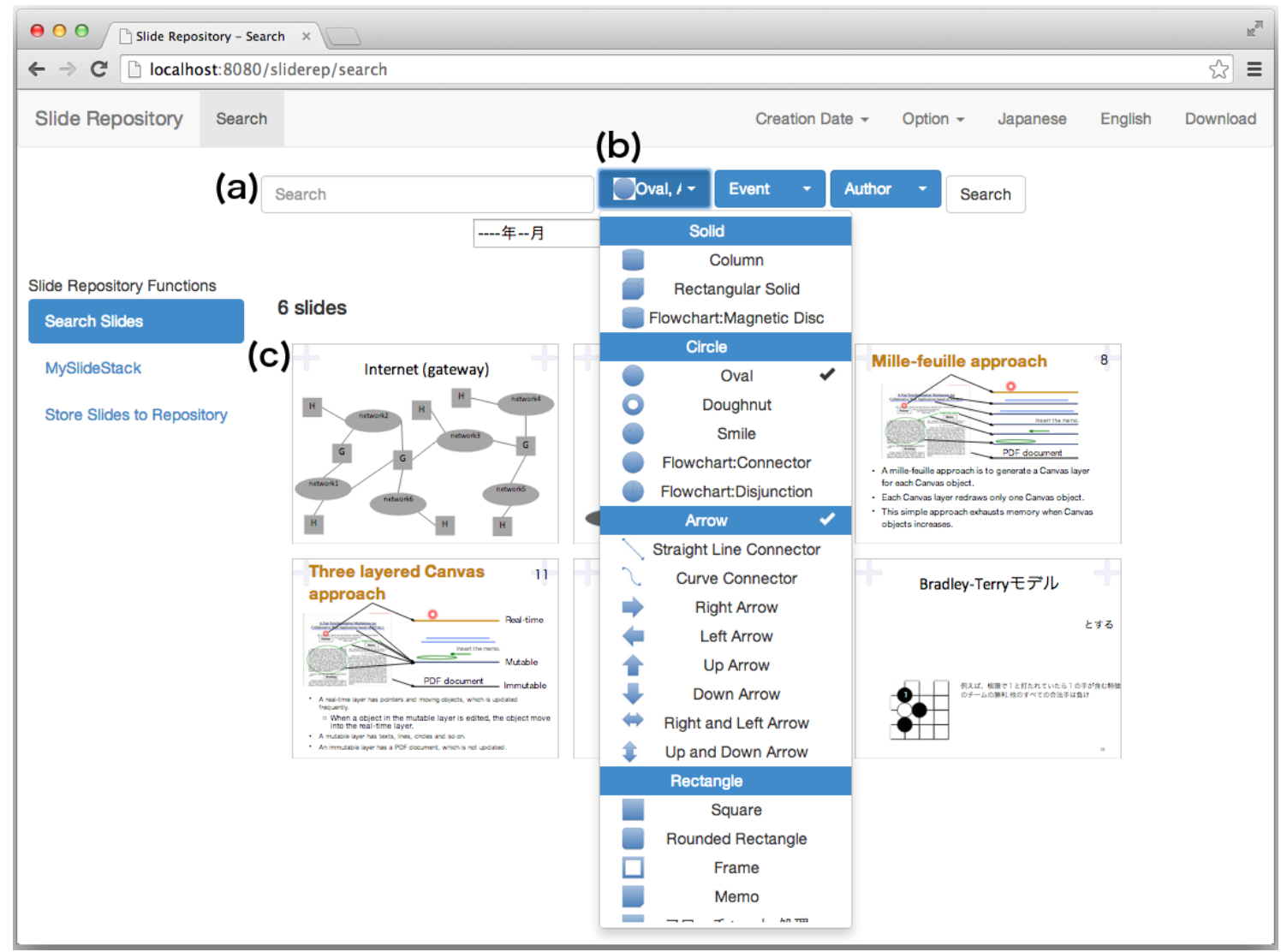

Fig. 5 Search interface for slide repository. 
which are defined by PowerPoint. The shapes are grouped by shape (e.g. "Solid", "Circle", "Arrow", "Rectangle", etc.). For example, authors select "Arrow", slides that contain "Right Arrow", "Left Arrow", "Up Arrow", or others are searched. The shape drop-down list supports multi selection with check boxes (e.g. select "Oval" and "Line"). The event list contains event names (e.g. seminar, ESKM2014, etc.).

\section{Evaluation}

\subsection{Experiment Method}

We conducted an experiment to confirm the effectiveness of Episodic Slide Retrieval based on Episodic Keyword Networks. We recruited 5 students (2 graduate and 3 undergraduate students) in our laboratory, who have an experience with creating presentation slides. Prior to conducting the experiment, we had two sessions for participants of 5 students, an introduction of the system and a briefing on the search purpose and tasks. The purpose of the introduction of the system was to inform participants of the functionality offered by Linked Data-based Slide Repository so that the participants can start using the system smoothly. Participants repeated the search task 20 times. The search task was structured as follows. Linked Data-based Slide Repository randomly showed participants a slide for a short time, the slide shown is the target slide that is supposed to be searched on the system. We gave them cognitive interference tasks. They searched the shown slide with Linked Data-based Slide Repository. Linked Data-based Slide Repository checked whether the found slide was the right one or not, and recorded the used queries and time spent on the search task.

\subsection{Experiment Setup}

Participants proceeded to perform the search task for 20 slides. The search task was structured as the following steps:

Linked Data-based Slide Repository shows one slide subject to be searched for 10 seconds on their web browsers and they remember the slide. They solve 5 additions of two-digit numbers as the cognitive interference task.

They try to search the slide. When they find it, they select the slide to check the answer. When they give up the search, they press a give-up button to indicate they could not find it successfully. Linked Data-based Slide Repository shows participants the result of the answer.

\subsection{Experiment Result}

Fig. 6 shows the percentage of slides found for the queries when participants found desired slides to all slides of the search task, and the percentage of time spent finding for the queries. "Keywords + Episodic Information" refers to percentage of the slides searched with queries of both keywords and episodic information (authors, shapes or the number of images). "Episodic Information" refers to percentage of the slide searched with queries of episodic information (both authors and the number of images, the number of images, or shapes). "Not Found" refers to the slides that participants did not find. Fig. 6(a) indicates that slide retrieval using episodic information searched half (7\%) of the slides that full-text search does not (14\%). The average length of strings was 8.5 , the average number of shapes was 0.25 , and the average number of images was 1.5 per a slide not found. It is assumed that the slides not found were slides containing a large image such as a screenshot.

\section{Discussion}

Our first study question was "Is Episodic Slide Retrieval based on Episodic Keyword Networks effective?" The experiment showed effectiveness of Episodic Slide Retrieval based on Episodic Keyword Networks with following three reasons. Firstly, Episodic Slide Retrieval based on Episodic Keyword Networks has a capability to search for slides that full-text search does not effectively work for. 
A: Keyword
C: Episodic Information

(a)

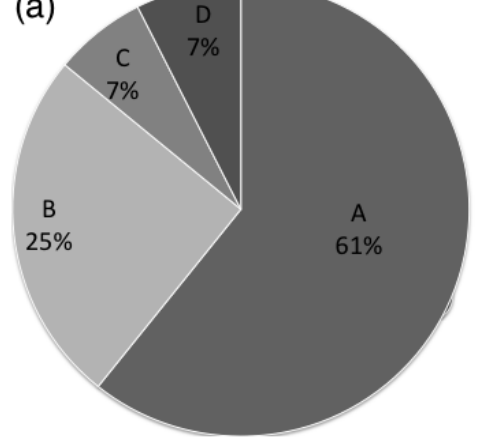

B: Keyword+Episodic Information

D: Not Found

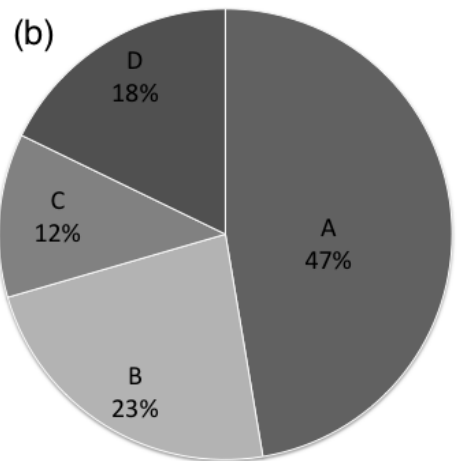

Fig. 6 (a) Percentage of slides found for the queries and (b) percentage of time spent finding for the queries.

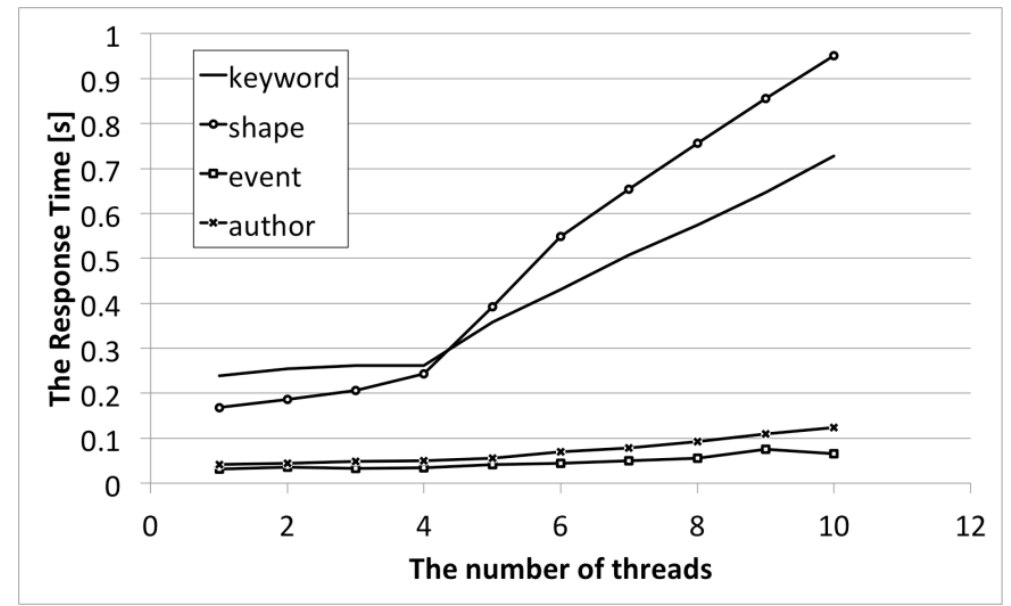

Fig. 7 Response time of the search.

Secondly, Episodic Slide Retrieval reduced the time spent in finding slides because participants spent about 3 minutes on average until giving up finding the slides on the full-text search, while the average time for search using episodic information was about 2 minutes. Thirdly, authors are able to search for some slides by refining search results for retry with the episodic information. Participants use both keywords and episodic information rather than only keywords. We observed that the shown slides reminded of participants author names, which indicates that authors retrieve slides with episodic information recalled from the slides. In order to confirm that the performance of Linked Data-based Slide Repository is practical for system operation, we evaluated the mean response time for 4 queries from sending a query to receiving the search results when from 1 to 10 authors search for slides at the same time.

Fig. 7 indicates that the mean response time with the number of threads. The response time for queries on an event that an author had is much shorter than for queries of part of description and shapes on the slides because nodes to search from "ressup:PresentationDoc" linked to the events and the authors are fewer than ones from "ressup:Slide" linked to the descriptions and shapes of contents of the slides. Hence, authors did not give up searching for slides on the ground of the high response speed.

Our second study question was "What method is appropriate for information retrieval using episodic information?" The answer is on a method using Linked Data. People have characteristics to associate memories with other memories. Similarly, it is effective to associate episodic information or slides 
with other episodic information for authors to be suggested.

Our third study question was "What is the way to collect episodic information?" Analyzing uploaded PowerPoint slides when the slides are transformed into SVG file automatically collects information of the shapes. On the other hand, authors inputting the information, which is time-consuming, collect information of events and authors. To address it, we plan to utilize a calendar application, a PowerPoint application and AppleScript. Three steps for the solution are as follows:

(1) To extract information of events from the calendar application with AppleScript; (2) To get the name of a PowerPoint file and the time stamp by monitoring PowerPoint when the user begins to give a presentation; (3) To associate the PowerPoint file with the event if the date of the time stamp is equal to the date of the event.

Our final study question was "What is appropriate methods of system implementation supporting slide reuse?" We divide the question into two points of view (user interface and slide expression). The user interface has various features supporting slide retrieval based on Episodic Keyword Networks. The user interface allowed authors to select event names, author names, or shape names from the drop-down lists because the authors perhaps do not know the shape names or remember current event names. In the shape drop-down list, especially, the shapes in the list are grouped by shape because authors accurately do not remember a shape name in desired slides. For example, they perhaps do not currently remember whether the shape is "square" or "rounded rectangle". Next, we expressed slides of PowerPoint as slides of SVG because of two problems about URIs of slides and a web application. There is also another way of slide expression that is a way with HTML and CSS. The way also solves two problems, but the expression does not have ability to reproduce slides equivalent to PowerPoint slides (e.g. the expression of shapes and animations). On the other hand, slide expression with SVG has ability to express the shapes and animations with <path> and <animate>.

Additionally, to pursue further efficiency in retrieving slides based on our conclusion that using episodic memories is the effective method to retrieve the desired slides from the repository, we had a discussion on expanding the structure of the Linked Data-based Slide Repository. For this discussion, we categorized the Linked Data in the repository as follows. The Linked Data-based Slide Repository consists of three tiers as the data model shown in Table 2.

As the Tier 1 Data, the information contained in as descriptions or attached to the presentation itself is assumed, such as the title and name of slide author, people listed as supported authors and contents in text, image and other non-text forms. As Tier 2 Data, the information directly related to the entire presentation and each slide are assumed, such as date and time of the presentation and each slide created and updated, presentation material's file and folder names, author's social networking handle and the mail account related to the slide contents. As Tier 3 Data, any obtainable background information related to the author's activities and associated with the Tire 1 and Tire 2 data having been retrieved from various external places, such as author's social media posts related to the slide creation activities, some social or private events on the slide creation date and time, and any other pieces of information to remind the authors of the specific slides.

On the experiment in this paper, we mainly focused on the Tier 1 and Tier 2 Data. It is our next step to focus on the Tier 3 Data to further explore the further slide-finding efficiency by expanding the episodic memories model in the repository taking in the links with the author-and-slide-related data and events having been post on the private and social media sites.

We assume this is achievable by making Linked Data-based Slide Repository support for automatically 
Table 2 Linked Data-based Slide Repository Tier Model.

\begin{tabular}{|c|c|c|}
\hline Tier & Definition & $\begin{array}{c}\text { Example } \\
\text { Query key Elements }\end{array}$ \\
\hline Tier 1 Data & $\begin{array}{l}\text { The native information attached to the } \\
\text { presentation itself is assumed, such as the } \\
\text { title and name of slide author, people } \\
\text { listed as supported authors and contents } \\
\text { in text, image and other non-text form }\end{array}$ & $\begin{array}{l}\text { Title: "AI for } \\
\text { Communication" } \\
\text { Author: "Tomohiro Iwasa" } \\
\text { Content: "... Episodic } \\
\text { Keyword Networks } \\
\text { asEpisodic Slide } \\
\text { Retrieval..." }\end{array}$ \\
\hline Tier 2 Data & $\begin{array}{l}\text { The information directly related to the } \\
\text { entire presentation and each slide are } \\
\text { assumed, such as date and time of the } \\
\text { presentation and each slide created and } \\
\text { updated, relevant file names, folder } \\
\text { names, author's social networking handle } \\
\text { and the mail account related to the slide } \\
\text { contents }\end{array}$ & $\begin{array}{l}\text { "AI for Communication": } \\
\text { "“Created on 2015/10/21", } \\
\text { "Tomohiro Iwasa", } \\
\text { "tiwasa@toralab.com", } \\
\text { "twitter:@tiwasa"\} }\end{array}$ \\
\hline Tier 3 Data & $\begin{array}{l}\text { Any background information related to } \\
\text { the author's activities and associated with } \\
\text { the Tire } 1 \text { and Tire } 2 \text { data having been } \\
\text { obtained from various external places, } \\
\text { such as author's social media posts related } \\
\text { to the slide creation activities, some social } \\
\text { or private events upon the slide creation } \\
\text { date and time, any of those piece of } \\
\text { information are to remind the authors of } \\
\text { the specific slides }\end{array}$ & $\begin{array}{l}\text { "Created on } \\
\text { 2015/10/21":"birthday" } \\
\text { "Slide Page 25":"AI for } \\
\text { Communication":"'Updated } \\
\text { on 2015/09/19":"Japan vs. } \\
\text { South Africa in the rugby } \\
\text { world cup 2015" } \\
\text { "AI for Communication": } \\
\text { "twitter:@tiwasa":"Posted on } \\
\text { 2015/08/30":"Now working } \\
\text { on the new proposal for } \\
\text { Company A" }\end{array}$ \\
\hline
\end{tabular}

Table 3 Tier query example.

\begin{tabular}{|c|l|l|}
\hline Tier & \multicolumn{1}{|c|}{ Query Keyword example } & \multicolumn{1}{c|}{ Result Example } \\
\hline Tier 1 Data & $\begin{array}{l}\text { "2015/10/21" \& "Episodic Slide } \\
\text { Retrieval" }\end{array}$ & $\begin{array}{l}\text { Slide Page 2, 3, 20, 21, 22 } \\
\text { File: AI for Communication }\end{array}$ \\
\hline Tier 2 Data & "Rugby 2015 Japan South Africa" & $\begin{array}{l}\text { Result: Slide Page } 25 \\
\text { File: AI for Communication }\end{array}$ \\
\hline Tier 3 Data & "Company A" & File: AI for Communication \\
\hline
\end{tabular}

making the links with the Tier 3 information for authors to allow to much more focus on the episodic memories to search the slides. Examples are shown in Table 3.

Furthermore, from usability standpoints, it is also a theme for us to consider in the extended study on the improvement from heuristic approach, updating the data in the repository by feedback from the user experience test and daily use of the system.

\section{Conclusions}

We proposed Linked Data-based Slide Repository and Episodic Keyword Networks. Linked Data-based Slide Repository is a repository searching, storing slides, and supporting the transformation of slides and presentation on a web browser. We have presented the 

the Episodic Keyword Networks

slide retrieval queries using Episodic Keyword Networks with examples. The experiment showed the effectiveness of Linked Data-based Slide Repository for the slide retrieval. Additionally, we discussed the improvement of the slide retrieval method for further effective retrieval of the required slides by expanding the structure of the Linked Data-based Slide Repository.

\section{References}

[1] Sharmin, M., Bergman, L., Lu, J., and Konuru, R. 2012. "On Slide-Based Contextual Cues for Presentation Reuse." In Proceedings of the 2012 ACM International Conference on Intelligent User Interfaces, 129-38.

[2] Mejova, Y., Schepper, K. D., Bergman, L., and Lu, J. 2011. "Reuse in the Wild: An Empirical and Ethnographic Study of Organizational Content Reuse." In Proceedings of the SIGCHI Conference on Human Factors in Computing Systems, 2877-86.

[3] Ravasio, P., Schar, S. G., and Krueger, H. 2004. "Pursuit of Desktop Evolution: User Problems and Practices with Modern Desktop Systems." ACM Transactions on Computer-Human Interaction 11 (2): 156-80.

[4] Johnson, M. K., Foley, M. A., Suengas, A. G., and Raye, C. L. 1988. "Phenomenal Characteristics of Memories for Perceived and Imagined Autobiographical Events."
Journal of Experimental Psychology General 117 (4): 371-6.

[5] Kalnikaite, V., Sellen, A., Whittaker, S., and Kirk, D. 2010. "Now Let Me See Where I Was: Understanding How Lifelogs Mediate Memory." In Proceedings of the SIGCHI Conference on Human Factors in Computing Systems, 2045-54.

[6] Fuller, M., Kelly, L., and Jones, G. J. F. 2008. “Applying Contextual Memory Cues for Retrieval from Personal Information Archives." In Proceedings of Personal Information Management, Workshop at CHI 2008.

[7] Jensen, C., Lonsdale, H., Wynn, E., Cao, J., Slater, M., and Dietterich, T. G. 2010. "The Life and Times of Files and Information: A Study of Desktop Provenance." In Proceedings of the 2010 ACM International Conference on Human Factors in Computing Systems, 767-76.

[8] Blanc-Brude, T., and Scapin, D. L. 2007. "What Do People Recall about Their Documents?: Implications for Desktop Search Tools." In Proceedings of the 12th International Conference on Intelligent User Interfaces, 102-11.

[9] Tanaka, S., Tezuka, T., Aoyama, A., Kimura, F., and Maeda, A. 2013. "Slide Retrieval Technique Using Features of Figures." In Proceedings of the 2013 International MultiConference of Engineers and Computer Scientists, 424-9.

[10] Prud'hommeaux, E., and Seaborne, A. 2005. "SPARQL Query Language for RDF." Accessed Mar. 19, 2014, from http://www.w3.org/TR/rdf-sparql- query/ot. 